\title{
Über Einwirkung von Baryumhydroxyd und von Natrium auf einige Aldehyde von
}

\section{Anton Lederer.}

Aus dem chemischen Laboratorium des Hofrathes Prof. Ad. Lieben an der k. k. Universität in Wien.

(Vorgelegt in der Sitzung am 25. April 1901.)

Das Verhalten der Fettaldehyde gegenüber verschiedenen condensierenden Agentien ist namentlich im hiesigen Laboratorium seit einer Reihe von Jahren Gegenstand eingehender Untersuchungen ${ }^{1}$ geworden. Anschließend an diese Arbeiten sind auch die in den folgenden Blättern beschriebenen Condensationsversuche ausgeführt.

\section{Condensationsversuche mit Baryumhydroxyd.}

Acetaldehyd, ${ }^{2}$ wie auch Crotonaldehyd, reagieren unter lebhafter Erwärmung mit concentrierten wässerigen Lösungen von Baryumhydroxyd. Indessen ermöglichte die in beiden Fällen erfolgte starke Verharzung nicht die Isolierung einheitlicher Producte.

Glatt hingegen verlief die Einwirkung des Baryumhydrats auf den Isobutyraldehyd. Hier konnte der bemerkenswerte Fall der Umwandlung des Aldehydes in Säure und Aldol festgestellt werden:

1 Lieben und $\mathrm{Z}$ eisel, Monatshefte für Chemie, $1880,818,840$; ebenda, 1883, 10, 531; ebenda, 1886, 53. Fos se k, Monatshefte für Chemie, 1881. 614; 1882,$622 ; 1883,660,663 ; 1884,119$. Fossek und Swoboda, Monatshefte für Chemie, 1890, 383. Li eb en und Schüler, 1896, 1897, 1898, 1899, 1900, 1901.

2 Tollens erhielt bei der Einwirkung von Baryumhydroxydlösung auf Acetaldehyd das Baryumsalz des sogenannten Aldehydgummis. Ber., 17, 660. 
$4\left\{\begin{array}{l}\mathrm{CH}_{3} \\ \mathrm{CH}_{3}\end{array}\right\rangle \mathrm{CH} \cdot \mathrm{CHO}+\mathrm{Ba}(\mathrm{OH})_{2}=$

$$
\left.=2\left\{\begin{array}{l}
\mathrm{CH}_{3} \\
\mathrm{CH}_{3}
\end{array}\right\rangle \mathrm{CH} \cdot \mathrm{CH}_{2} \mathrm{OH}+\left[\begin{array}{l}
\mathrm{CH}_{3} \\
\mathrm{CH}_{3}
\end{array}\right\rangle \mathrm{CHCOO}\right]_{2} \mathrm{Ba} .
$$

Bei Einhaltung der folgenden Versuchsbedingungen war die Bildung von Alkohol und Säure eine nahezu quantitativ nach dieser Gleichung verlaufende Reaction: $25 \mathrm{~g}$ acetonfreien Isobutyraldehyds wurden mit $15 \mathrm{~g}$ Baryumhydroxyd (d. i. ungefähr auf 4 Molecüle Aldehyd 1 Molecül Barythydrat). und $30 \mathrm{~g}$ Wasser in ein Rohr eingeschmolzen und 14 Stunden auf $150^{\circ}$ erhitzt. Beim Öffnen der Einschmelzröhre zeigte sich, dass die obere Schichte, die ursprünglich aus Isobutyraldehyd bestand, nunmehr kaum Aldehydgeruch aufwies; desgleichen war die untere Schichte, die ursprünglich die Baryumhydroxydlösung enthielt, verändert, wie man aus der jetzt nur ganz schwach alkalischen Reaction dieser wässerigen Lösung vermuthen konnte. Der gesammte Röhreninhalt wurde der Destillation im Wasserdampfstrome unterzogen, solange noch riechende Destillate erhalten wurden. Die vereinigten Destillate wurden mit Pottasche gesättigt, die aufschwimmende Schichte abgehoben, über festem Kaliumcarbonat getrocknet und schließlich fractioniert. Ohne einen nennenswerten Vorlauf destillierte die Hauptmenge von 107 bis $109^{\circ}$.

Schon dieser Siedepunkt, wie auch der Geruch, bewiesen zweifellos, dass reiner Isobutyralkohol vorliegt. Desgleichen bestätigte dies die nach der Methode Bleier-Kohn ausgeführte Moleculargewichtsbestimmung.

$0.0235 \mathrm{~g}$ Substanz lieferten, im Dampfe des siedenden Toluols vergast, eine Druckerhöhung von $289 \mathrm{~mm}$ Paraffinöl (Constante für Toluol $=907)$. Daraus berechnetes Moleculargewicht:

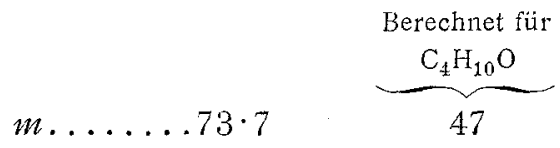

Der nach der Wasserdampfdestillation zurückgebliebene Rückstand, der das Baryums $\mathrm{l} z$ der entstandenen Säure entChemie-Heft Nr. 6. 
halten musste, wurde mit Phosphorsäure stark angesäuert, wobei eine ölige Säure zur Abscheidung kam, die ausgeäthert wurde. Die ätherische Lösung wurde über entwässertem Natriumsulfat getrocknet. Der nach Entfernung des Äthers resultierende Rückstand gieng bei der Fractionierung von 155 bis $156^{\circ}$ über. Zum Zwecke der vollständigen Identificierung wurde die Säure durch Kochen mit Silbercarbonat bis zum Verschwinden der sauren Reaction in ihr Silbersalz verwandelt. Aus der vom überschüssigen Silbercarbonat heiß filtrierten Lösung fiel beim Erkalten ein rein weißes, gut krystallisiertes. Silbersalz aus, das vacuumtrocken zur Analyse gelangte.

I. $0 \cdot 3375 \mathrm{~g}$ Silbersalz ließen $0 \cdot 18675 \mathrm{~g}$ Silber.

II. $0 \cdot 3525 g$ Silbersalz ließen $0 \cdot 1950 \mathrm{~g}$ Silber.

In 100 Theilen:

$\overbrace{\text { I. }}^{\text {Ag } \ldots \ldots \ldots 55 ; 33} \underbrace{\text { Gefunden }}_{\text {II. }} \underbrace{\begin{array}{c}\text { Berechnet für } \\ \mathrm{C}_{4} \mathrm{H}_{7} \mathrm{O}_{2} \mathrm{Ag}\end{array}}_{55 \cdot 31}$

Diese Bestimmungen beweisen gleichfalls, dass die Säure Isobuttersäure ist.

Die gleichzeitige Bildung von Alkohol und Säure aus 2 Molecülen Aldehyd bei Einwirkung von Alkali als quantitativ verlaufende Reaction ist bisher nur bei jenen Aldehyden beobachtet worden, die an dem der Aldehydgruppe benachbarten Kohlenstoffatom ( $\alpha$-Kohlenstoffatom) kein Wasserstoffatom enthalten. Hierher gehören also Aldehyde cyclischer Verbindungen, bei denen ein Kernwasserstoffatom durch die Aldehydgruppe ersetzt ist (z.B. der Benzaldehyd-Reaction von Cannizaro-, Furfurol), ferner der $\alpha$-Oxyisobutyraldehyd, wie erst jüngst Franke, ${ }^{1}$ und der 2-Dimethyl-3-Oxypropionaldehyd, wie neuerdings Wessely² gezeigt hat. Auch das Oxymethylen liefert nach den Angaben von Tollen $\mathrm{s}^{3}$ bei Einwirkung von Magnesia Ameisensäure und Methylalkohol. Doch ist zu bemerken, dass.

1 Monatshefte für Chemie, 1900, 1122.

2 Monatshefte für Chemie, 1901, 66.

3 Berl. Ber., 16, 919. 
das Verhalten des Formaldehyds bei Condensationsvorgängen überhaupt ein anomales ist.

Es muss demnach im Lichte dieser Auffassung besonders auffällig und bemerkenswert erscheinen, dass der Isobutyraldehyd, wie wohl er am $\alpha$-Kohlenstoffatom ein Wasserstoffatom enthält, und daher auch im Sinne der Regel von Lieben und Zeisel glatt zu Isobutyraldol condensierbar ist, gerade durch Erhitzen mit Barythydrat quantitativ in Isobutylalkohol und Isobuttersäure überführt werden kann. ${ }^{1}$

Auf das Valeral wirkte Ätzbaryt nur condensierend ein.

Reines Valeral wurde mit Barythydrat (auf 4 Molecüle Valeral 1 Molecül Baryumhydroxyd) und Wasser (nahezu die gleiche Gewichtsmenge wie das Baryumhydroxyd) in ein Rohr eingeschmolzen. und 10 Stunden auf $130^{\circ}$ erhitzt. Hiebei hatte sich die ursprüngliche Aldehydschichte gelb gefärbt und einen durchdringenden, stechenden Geruch angenommen. Hingegen war das Baryumhydroxyd anscheinend zum größten Theile unverändert geblieben, wie man aus der stark alkalischen Reaction der wässerigen Lösung schließen konnte. Der gesammte Röhreninhalt wurde mit Wasserdampf destilliert, bis alles Öl übergangen war. Durch Ausschütteln mit Äther wurde es dem Destillate entzogen. Bei der nach Entfernung des Äthers im $\mathrm{CO}_{2}$-Strome vorgenommenen Fractionierung destillierte die Hauptmenge von 186 bis $189^{\circ}$ (uncorr).

1 Anmerkung. Die Beobachtung Hern Le derers steht in vollkommener Übereinstimmung mit der kürzlich von mir ausgesprochenen Ansicht (Monatshefte für Chemie, 1901, S. 308), dass die Spaltbarkeit in Säure und Alkohol eigentlich allen Aldehyden zukommt, dass sie aber nicht immer beobachtet werden kann, weil in vielen Fällen andere Reactionen sich rascher vollziehen. Ich habe hinzugefügt: *Wenn es gelingt, die Aldolbildung hintanzuhalten, so scheint es mir durchaus nicht ausgeschlossen, dass auch bei Aldehyden, die nicht zur III. Gruppe gehören, eine Spaltung in Säure und Alkohol sich erzielen lassen wird «.

Dieser Fall ist hier eingetreten. Durch das Erhitzen auf $150^{\circ}$ wird die Aldolbildung verhindert und wird entstandenes Isobutyraldol in Aldehyd zurückverwandelt. Der Isobutyraldehyd. unter dem Einflusse von Baryumhydroxyd erleidet die Spaltung in Isobutyralkohol und Isobuttersäure, wobei als erstes Condensationsproduct vorübergehend der isobuttersaure Isobutylester entstehen dürfte.

Ad. Lieben. 
Der Siedepunkt, die Bromadditionsfähigkeit und der Geruch ließen keinen $Z$ weifel obwalten, dass die Substanz das von L. Kohn ${ }^{1}$ studierte ungesättigte Condensationsproduct des Valerals ( $\alpha$-Isopropyl- $\beta$-Isobutylacrolein) repräsentiert. Auch die Moleculargewichtsbestimmung bewies dies.

$0.0420 \mathrm{~g}$ Substanz bewirkten, im evacuierten, mit siedendem Toluol geheizten Raume vergast, eine Druckerhöhung von $250 \mathrm{~mm}$ Paraffinöl (Constante $=907$ ).

Daraus berechnetes Moleculargewicht:

$$
m \ldots \ldots \ldots 2 \cdot 3 \quad \frac{\begin{array}{c}
\text { Berechnet für } \\
\mathrm{C}_{10} \mathrm{H}_{18} \mathrm{O}
\end{array}}{154}
$$

Die nach der Wasserdampfdestillation zurückgebliebene Lösung wurde mit Phosphorsäure angesäuert, und die geringe Menge der abgeschiedenen öligen Säure mit Äther aufgenommen. Nach Entfernung desselben blieb ein vermuthlich aus Isovaleriansäure bestehender Rückstand, der sich infolge seiner geringen Menge allerdings jeder weiteren Untersuchung entzog.

\section{Einwirkung von metallischem Natrium ${ }^{2}$ auf Isobutyraldehyd und auf Valeral.}

In acetonfreien Isobutyraldehyd, der sich in einem mit Rückfussskühler verbundenen Rundkolben befand, wurde Natrium in linsengroßen Stücken in nicht zu rascher Folge eingetragen, indem mit dem Zusatze eines neuen Natriumstückchens so lange gewartet wurde, bis alles eingebrachte Metall aufgebraucht war.

Die Reaction wird auch unter diesen Versuchsbedingungen allmählig eine ziemlich heftige, so dass äußere Kühlung sich zweckmäßig erwies. Nach einiger Zeit beginnt die Einwirkung sich zu mäßigen; die Flüssigkeit trübt sich und nimmt eine ölige Consistenz an. Schließlich bleibt das eingetragene Natrium

1 Monatshefte für Chemie, 1896, 126 u. f.

2 Freer, Ann., 293, 327. Church, Ann., 128, 295. Beckmann und Paul, Ann., 266, 25. 
unangegriffen. Nụn wurde Äther zugesetzt, die ätherische Lösung mit Wasser bis zum Verschwinden der alkalischen Reaction gewaschen und hierauf mit geschmolzenem Chlorcalcium getrocknet. Das vom Äther befreite Product lieferte, im Vacuum destilliert, nach einem kaum nennenswerten. Vorlauf, der den Geruch nach Isobutylalkoho ${ }^{1}$ zeigte, eine bei $17 \mathrm{~mm}$ von 135 bis $137^{\circ}$ siedendẻ Hauptfraction.

Die Elementaranalysen ergaben die Zahlen:

I. $0 \cdot 170 \mathrm{~g}$ Substanz lieferten $0.41375 \mathrm{~g} \mathrm{CO}_{2}$ und $0.1745 \mathrm{~g}$ $\mathrm{H}_{2} \mathrm{O}$.

I. $0.232 \mathrm{~g}$ Substanz lieferten $0.5650 \mathrm{~g} \mathrm{CO}_{2}$ und $0.2370 \mathrm{~g} \mathrm{H}_{2} \mathrm{O}$. In 100 Theilen:

\begin{tabular}{|c|c|c|c|}
\hline & \multicolumn{2}{|c|}{ Gefunden } & \multirow{2}{*}{$\begin{array}{l}\text { Berechnet für } \\
\mathrm{C}_{12} \mathrm{H}_{24} \mathrm{O}_{3}\end{array}$} \\
\hline & I. & II. & \\
\hline C. . & $.66 \cdot 37$ & $66 \cdot 41$ & $66 \cdot 66$ \\
\hline $\mathrm{H} \ldots \ldots$ & $.11 \cdot 40$ & $11 \cdot 11$ & $11 \cdot 11$ \\
\hline
\end{tabular}

Die Ergebnisse der Elementaranalysen, der Siedepunkt und die anderen Eigenschaften der Substanz deuteten auf ihre Identität mit dem von $\mathrm{Brauchbar}$ und L. Kohn ${ }^{2}$ untersuchten Octoglycolisobutyrat hin.

Um dies nun in unzweifelhafter Weise darzuthun, war die Verseifung zu Glycol und Isobuttersäure erforderlich.

Demnach wurden $8 g$ der Substanz mit überschüssigem alkoholischen Kali eine Stunde am Rückflusskühler im Wasserbade in mäßigem Sieden erhalten. Dann wurde durch Einleiten von $\mathrm{CO}_{2}$ neutralisiert und der Alkohol aus dem Wasserbade abdestilliert. Der Rückstand wurde mit Wasser bis zur Lösung des Kaliumcarbonates versetzt und dann ausgeäthert. Nach dem Verjagen des Äthers wurde im Vacuum destilliert. Unter einem Drucke von $22 \mathrm{~mm}$ gieng constant von 144 bis $145^{\circ}$ ein zähes, wasserklares Liquidum über, das in der Vorlage zu einem compacten Krystallkuchen erstarrte, der, abgepresst und aus Äther umkrystallisiert, den Schmelzpunkt 51 bis $52^{\circ}$ zeigte.

1 Im vorliegenden Falle verdankt der Isobutyralkohol vermuthlich seine Entstehung der reducierenden Wirkung des metallischen Natriums auf Isobutyraldehyd in Gegenwart yon etwas Feuchtigkeit.

2 Monatshefte für Chemie, 1898,16 u. f. 
Auch die Elementaranalyse führte zu Zahlen, die mit denl für das Fossek'sche Glycol berechneten stimmten.

$0.225 \mathrm{~g}$ Substanz lieferten bei der Verbrennung $0.5475 \mathrm{~g} \mathrm{CO}_{2}$ und $0 \cdot 2455 g_{2} \mathrm{H}_{2} \mathrm{O}$.

In 100 Theilen:

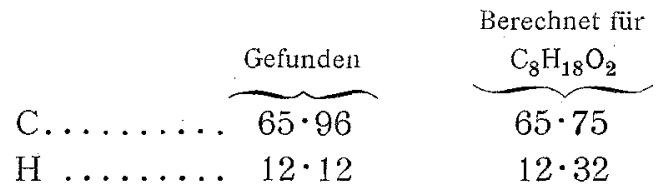

Die vom Glycol durch Ausäthern befreite, stark alkalische Flüssigkeit wurde mit mäßig concentrierter Schwefelsäure angesäuert, und die frei gemachte Säure mit Äther ausgeschüttelt. Nach Entfernung des Äthers wurde sie mit überschüssigem Calciumcarbonat und Wasser unter Rückfluss bis zum Verschwinden der sauren Reaction gekocht. Die heiß vom kohlensauren Kalk abfiltrierte Lösung schied beim Erkalten ein krystallinisches Calciumsalz ab, das durch die Analyse als Calciumisobutyrat erkannt wurde.

$0 \cdot 21375 g$ des im Toluolbade scharf getrockneten Salzes lieferten, bis zur Gewichtsconstanz geglüht, $0.05625 \mathrm{~g}$ $\mathrm{CaO}$.

In 100 Theilen:

$$
\underbrace{\begin{array}{l}
\text { Berechnet für } \\
\left(\mathrm{C}_{4} \mathrm{H}_{7} \mathrm{O}_{2}\right)_{2} \mathrm{Ca}
\end{array}}_{18 \cdot 69}
$$$$
\mathrm{Ca} . . . \ldots \overbrace{18 \cdot 79}
$$

Die Bildung des Isobuttersäureesters des Octoglycols erscheint leicht erklärlich, wenn man annimmt, dass durch die geringe Menge der dem Aldehyd stets anhaftenden Feuchtigkeit aus dem Natrium zunächst Natriumhydroxyd gebildet wird, das zunächst Aldolcondensation und dann weiterhin die Estercondensation einzuleiten imstande sein müsste.

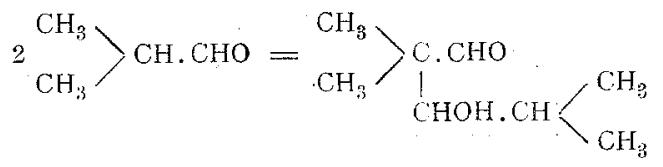


II.

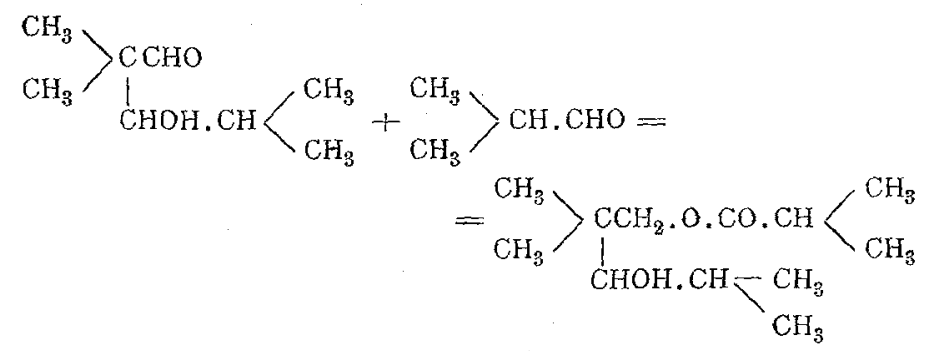

Thatsächlich haben denn auch Franke und L. Kohn ${ }^{1}$ durch Einwirkung geringer Mengen von alkoholischem Natron auf Isobutyraldehyd diesen Ester erhalten.

Es war somit jetzt zum Zwecke der Bestätigung der Richtigkeit dieser Auffassung über die Einwirkung des metallischen Natriums auf den Isobutyraldehyd $z u$ zeigen, dass wirklich jene geringen Mengen von Natriumhydroxyd glatte Estercondensation bewirken können.

Nun hatte sich aus meinen Versuchen ergeben, dass durchschnittlich auf $35 \mathrm{~g}$ Isobutyraldehyd ungefähr $0.7 \mathrm{~g}$ Natrium verbraucht werden; diese Menge Natrium entspricht $1.21 \mathrm{~g}$ Natriumhydroxyd. Demgemäß wurden $35 \mathrm{~g}$ Isobutyraldehyd mit $1 \cdot 21$ g gepulvertem Natriumhydroxyd, das, um die Heftigkeit der Reaction zu mäBigen, in mehreren Antheilen eingetragen wurde, geschüttelt. Dabei konnte genau der gleiche Verlauf der Einwirkung wie bei der Anwendung von metallischem Natrium constatiert werden. Das Reactionsproduct wurde mit Äther verdünnt, die ätherische Lösung durch Waschen mit Wasser vom anhaftenden Alkali befreit und dann über geschmolzenem Chlorcalcium getrocknet. Nach dem Abdestillieren des Äthers gieng das Product unter einem Drucke von 16 bis $17 \mathrm{~mm}$ constant von 130 bis $133^{\circ}$ über.

Die Analyse ergab:

$0.2625 \mathrm{~g}$ Substanz lieferten $0.640 \mathrm{~g} \quad \mathrm{CO}_{2}$ und $0.26575 \mathrm{~g}$ Wasser.

In 100 Theilen:

Berechnet für

$$
\begin{aligned}
& \text { C ...... } \frac{\text { Gefunden }}{66 \cdot 50} \\
& \underbrace{\mathrm{C}_{12} \mathrm{H}_{24} \mathrm{O}_{3}}_{66 \cdot 66} \\
& \text { H ....... } 11 \cdot 25 \\
& 11 \cdot 11
\end{aligned}
$$

1 Monatshefte für Chemic, 19, S. 361. 
Es ist somit auch hier in glatter Reaction das Octoglycolisobutyrat entstanden. Hingegen zeigte der Vorlauf von der Vacuumdestillation des mit Natriumhydroxyd dargestellten Esters nicht den Geruch nach Isobutylalkohol, wie aus der Abwesenheit eines reducierenden Agens erklärlich erscheint. Wohl aber konnte bei der Einwirkung von Natriumbydroxyd Polymerisation einer geringen Aldehy dmenge beobachtet werden.

Schließlich erwähne ich noch, dass die Einwirkung des. metallischen Natriums auf den Isovaleraldehyd ganz in der gleichen Weise verlief wie beim Isobutyraldehyd. Ich erhielt hiebei im Gegensatze zu Borodine, ${ }^{1}$ der einen complexen Reactionsverlauf beschreibt, in glatter Reaction das von L. Kohn ${ }^{2}$ beschriebene Polymere des Valerals, das nach bisher unveröffentlichten, im hiesigen Laboratorium ausgeführten Untersuchungen die Constitution des Isovaleriansäureesters (Siedepunkt $150^{\circ}$ bei $16 \mathrm{~mm}$ Druck) des entsprechenden Glycols besitzt. Die Bildung dieses Körpers ist deshalb bemerkenswert, weil daraus hervorgeht, dass das Valeraldol, trotzdem es besondere Tendenz ${ }^{3}$ zur Wasserabspaltung zeigt, doch mit einem Molecül Valeral unter gewissen Bedingungen Estercondensation einzugehen imstande ist, wie dies auch bei der Einwirkung des alkoholischen Kalis auf den Isovaleraldehyd erfolgt.

Zum Schlusse dieser Ausführungen erlaube ich mir, meinem hochverehrten Lehrer, Herrn Hofrath Prof. Dr. Adolf Lieben, für sein liebenswürdiges Entgegenkommen und die vielen, wertvollen Rathschläge, die er mir im Verlaufe meiner Árbeiten zukommen ließ, meinen ergebensten Dank auch an dieser Stelle auszusprechen.

1 J. pr. Ch., 93, 413 (1865). Jahresbericht, 1864, 338. Berl. Ber., 5, 481.

2 Monatshefte für Chemie, 1896, 146 u. f.

3 Monatshefte für Chemie, 1897. 